\title{
ENDOSCOPIC TRANSORAL MEDIALIZATION VERSUS THYROPLASTY TYPE I OF THE VOCAL FOLDS IN PATIENTS WITH GLOTTAL INSUFFICIENCY
}

Mohamed Hossam EI-Deen Mostafa Hassen Thabet, Mostafa Magdy Elsaied Donia, Reham Mohamed Elmaghraby

\section{Department of Otorhinolaryngology, Faculty of Medicine, Alexandria University}

\section{Introduction}

Incomplete vocal fold adduction during phonation causes patients to complain of difficulty in phonation, especially when they are competing with back-ground noise. Patients with unilateral vocal fold paralysis typically complain of breathy dysphonia, vocal fatigue, and aspiration of liquids. The treatment of unilateral vocal cord paralysis has a long history, marked by technical innovations and improvements. These methods typically use endoscopic injection or implants to augment the volume of the vocal fold. In medialization thyroplasty, the position of the vocal cord is medialized by an external approach in which a permanent implant is placed through a thyroid cartilage window intraoperatively, often with conscious sedation and intra-operative voice analysis. Medialization thyroplasty using various implants, commonly expanded polytetrafluoroethylene (E-PTFE) or Silastic, is seemed the gold standard treatment for large glottic gaps and is the mainstay surgical intervention for unilateral vocal cord palsy. Medialization thyroplasty is a widely accepted surgical procedure for improving insufficient glottal closure in patients with unilateral recurrent laryngeal nerve paralysis.

\section{Aim of the work}

The aim of this study was to compare between the endoscopic medialization of the vocal folds using autogenous nasal cartilage and thyroplasty type I using silastic block \pm arytenoid adduction if needed in patients with glottal insufficiency.

\section{Patients and methods}

The study was carried on twenty patients having glottal insufficiency attending the outpatient clinic, Otorhinolaryngology Department, Main Alexandria University. Group I were subjected to endoscopic medicalization of the vocal folds using autogenous nasal cartilage under general anaethesia.

Group II were subjected to thyroplasty type I using silastic block \pm arytenoid adduction if needed under local anaethesia if possible. Each patient was subjected to the following assessment: Patient interview including complete history taking and analysis of the patient's symptoms
Full ENT examination of the patient. Auditory perceptual assessment (APA) using the modified GRBAS scale. Laryngovideostroboscopy was done for augmentation, documentation of the visual assessment of the vocal organ assessment of the vocal fold vibration and the size of the glottal gap Acoustic analysis was done in order to assess the average fundamental frequency, jitter $\%$, shimmer $\%$, noise to harmonic ratio. All patients will be subjected to the protocol of assessment preoperatively, and six months postoperatively.

\section{Results}

Table (1): Comparison between the two studied groups according to grade of dysphonia.

\begin{tabular}{|c|c|c|c|c|c|c|}
\hline \multirow{2}{*}{ Grade of dysphonia } & \multicolumn{2}{|c|}{$\begin{array}{l}\text { Group I } \\
(n=10)\end{array}$} & \multicolumn{2}{|c|}{$\begin{array}{l}\text { Group II } \\
(n=10)\end{array}$} & \multirow{2}{*}{$x^{2}$} & \multirow{2}{*}{ p } \\
\hline & No. & $\%$ & No & $\%$ & & \\
\hline \multicolumn{7}{|l|}{ Pre-operative } \\
\hline Mild & 0 & 0.0 & 0 & 0.0 & \multirow{3}{*}{0.800} & \multirow{3}{*}{0.371} \\
\hline Moderate & 6 & 60.0 & 4 & 40.0 & & \\
\hline Severe & 4 & 40.0 & 6 & 60.0 & & \\
\hline \multicolumn{7}{|l|}{ Post-operative } \\
\hline Mild & 6 & 60.0 & 8 & 80.0 & \multirow{4}{*}{0.952} & \multirow{4}{*}{$\begin{array}{c}F E p= \\
0.628\end{array}$} \\
\hline Moderate & 4 & 40.0 & 2 & 20.0 & & \\
\hline Severe & 0 & 0.0 & 0 & 0.0 & & \\
\hline $\mathrm{MH}\left(\mathrm{p}_{0}\right)$ & \multicolumn{2}{|c|}{$19.0^{*}\left(0.002^{*}\right)$} & \multicolumn{2}{|c|}{$19.0^{*}\left(0.003^{*}\right)$} & & \\
\hline
\end{tabular}

\section{$\chi^{2}$ : Chi square test}

FE: Fisher Exact

MH: Marginal

Homogeneity Test

p: $p$ value for comparing between the studied groups

$p_{0}:$ p value for comparing between
$*$ : Statistically significant at $\mathrm{p} \leq 0.05$
Table (2):Comparison between pre and post in each group according to jitter.

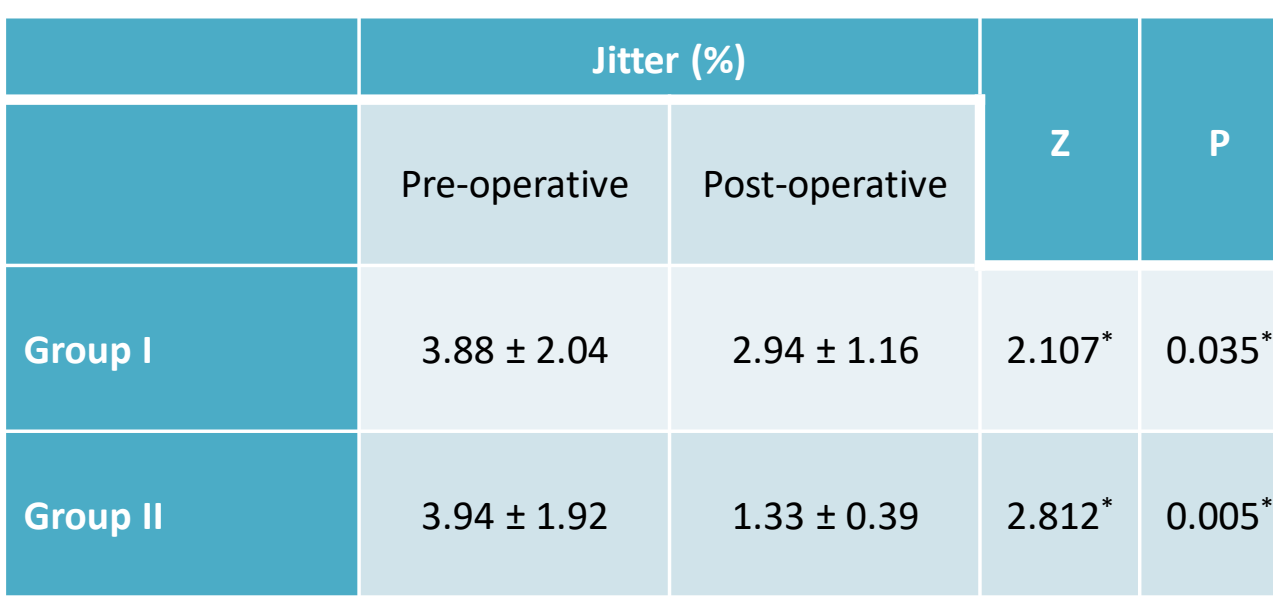

Z: Wilcoxon signed ranks test

$\mathrm{p}$ : $\mathrm{p}$ value for comparing between Pre-operative and Post-operative in $\mathrm{p}: \mathrm{p}$ value for
each group

*: Statistically significant at $\mathrm{p} \leq 0.05$

\section{Conclusion}

This method of medialization of the vocal fold using autologous nasa septal cartilage and endolaryngeal microsurgery by transoral approach for the treatment of high vagal paralysis seems to be safe, quick and efficient phonosurgical procedure for treatment of patients with unilateral vocal cord paralysis

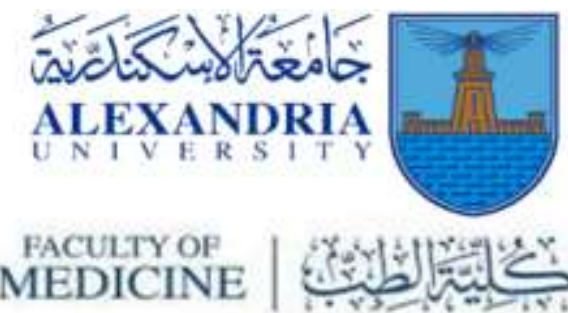

2021@Alexandria Faculty of Medicine CC-BY-NC 